\title{
Mass accretion rates from multiband photometry in the Carina Nebula: the case of Trumpler $14^{\star}$
}

\author{
G. Beccari ${ }^{1}$, G. De Marchi ${ }^{2}$, N. Panagia ${ }^{3,4,5}$, E. Valenti ${ }^{6}$, G. Carraro ${ }^{1}$, M. Romaniello ${ }^{6}$, \\ M. Zoccali ${ }^{7,8}$, and C. Weidner ${ }^{9,10}$
}

${ }^{1}$ European Southern Observatory, Av. Alonso de Cordova, 3107, 19001 Casilla, Santiago, Chile e-mail: gbeccari@eso.org

${ }^{2}$ ESA, Space Science Department, Keplerlaan 1, 2200 AG Noordwijk, The Netherlands

Space Telescope Science Institute, Baltimore, MD 21218, USA

4 INAF-NA, Osservatorio Astronomico di Capodimonte, Salita Moiariello 16, 80131 Napoli, Italy

5 Supernova Ltd, OYV \#131, Northsound Rd., Virgin Gorda VG1150, Virgin Islands, UK

${ }^{6}$ European Southern Observatory, Karl-Schwarzschild-Strasse 2, 85748 Garching bei München, Germany

7 Instituto de Astrofísica, Pontificia Universidad Catlica de Chile, Av. Vicua Mackenna 4860, 782-0436 Macul, Santiago, Chile

${ }^{8}$ Millennium Institute of Astrophysics, Av. Vicua Mackenna 4860, 782-0436 Macul, Santiago, Chile

9 Instituto de Astrofsica de Canarias, Calle Va Lactea s/n, 38205 La Laguna, Tenerife, Spain

10 Dept. Astrofisica, Universidad de La Laguna (ULL), 38206 La Laguna, Tenerife, Spain

Received 27 April 2014 / Accepted 12 September 2014

\section{ABSTRACT}

\begin{abstract}
We present a study of the mass accretion rates of pre-main sequence (PMS) stars in the cluster Trumpler 14 (Tr 14) in the Carina Nebula. Using optical multiband photometry we were able to identify 356 PMS stars showing $\mathrm{H} \alpha$ excess emission with equivalent width $E W(\mathrm{H} \alpha)>20 \AA$. We interpret this observational feature as an indication that these objects are still actively accreting gas from their circumstellar medium. From a comparison of the HR diagram with PMS evolutionary models we derive ages and masses of the PMS stars. We find that most of the PMS objects are younger than $10 \mathrm{Myr}$ with a median age of $\sim 3$ Myr. Surprisingly, we also find that $\sim 20 \%$ of the mass accreting objects are older than 10 Myr. For each PMS star in Trumpler 14 we determine the mass accretion rate $\left(\dot{M}_{\text {acc }}\right)$ and discuss its dependence on mass and age. We finally combine the optical photometry with near-IR observations to build the spectral energy distribution (SED) for each PMS star in Tr 14. The analysis of the SEDs suggests the presence of transitional discs in which a large amount of gas is still present and sustains accretion onto the PMS object at ages older than 10 Myr. Our results, discussed in light of recent recent discoveries with Herschel of transitional discs containing a massive gas component around the relatively old PMS stars TW Hydrae, 49 Ceti, and HD 95086, support a new scenario $\mathrm{n}$ which old and evolved debris discs still host a significant amount of gas.
\end{abstract}

Key words. accretion, accretion disks - stars: pre-main sequence

\section{Introduction}

Mass accretion is a phenomenon that drives the evolution of several astrophysical objects. It is well known that low-mass stars in the pre-main sequence (PMS) stage increase in mass through mass accretion from a circumstellar disc (e.g. Lynden-Bell \& Pringle 1974; Appenzeller \& Mundt 1989; Bertout 1989). Moreover, the process of accretion is the power source driving the luminosities for a wide range of binary systems containing accreting white dwarfs and neutron stars like cataclysmic variables and millisecond pulsars, black holes $(\mathrm{BH})$, gammaray bursts, and supermassive BHs in active galactic nuclei (see Fender et al. 2009). In an accreting star this process leaves characteristic signatures in its spectrum mostly due to $\mathrm{H}^{+}$recombinations. Thus, one expects an excess in the ultraviolet (UV) continuum, compared to stars of equal spectral type, (e.g. Gullbring et al. 1998; Rigliaco et al. 2011), as well as a strong emission in recombination lines (e.g. $\mathrm{H} \alpha, \mathrm{Pa} \beta$, and $\mathrm{Br} \gamma$ ) from the gas ionized in the shock.

De Marchi et al. (2010, hereafter DM10) have presented a method of reliably identifying PMS objects actively undergoing mass accretion in star forming regions using photometry. Briefly, the method combines $V$ and $I$ broadband photometry with narrowband $\mathrm{H} \alpha$ imaging to identify all stars with excess $\mathrm{H} \alpha$ emission and to measure their associated $\mathrm{H} \alpha$ emission equivalent width $E W(\mathrm{H} \alpha)$, the $\mathrm{H} \alpha$ luminosity, and the mass accretion rate, regardless of their age. The ability of this method to measure accurate $E W(\mathrm{H} \alpha)$ has been discussed thoroughly and justified by DM10 and verified observationally for stars towards IC1396 by Barentsen et al. (2011). In their Fig. 5 these authors show an excellent correlation between the photometric and spectroscopic measures of $E W(\mathrm{H} \alpha)$ for emission stronger than $10 \AA$. The DM10 method was recently applied to the study of PMS objects in a number of star-burst clusters, namely NGC 3603 in the Galaxy (Beccari et al. 2010), NGC 346 and NGC 602 in the Small Magellanic Clouds (see De Marchi et al. 2011a, 2013a), 30 Doradus and three active star forming regions in the Large Magellanic Cloud (see De Marchi et al. 2011b; Spezzi et al. 2012).

In this paper we use the same method to study the PMS stars in the young star-burst cluster Trumpler 14 (Tr 14) in the Carina Nebula. Tr 14 is a young cluster, about $1-3$ Myr old, 


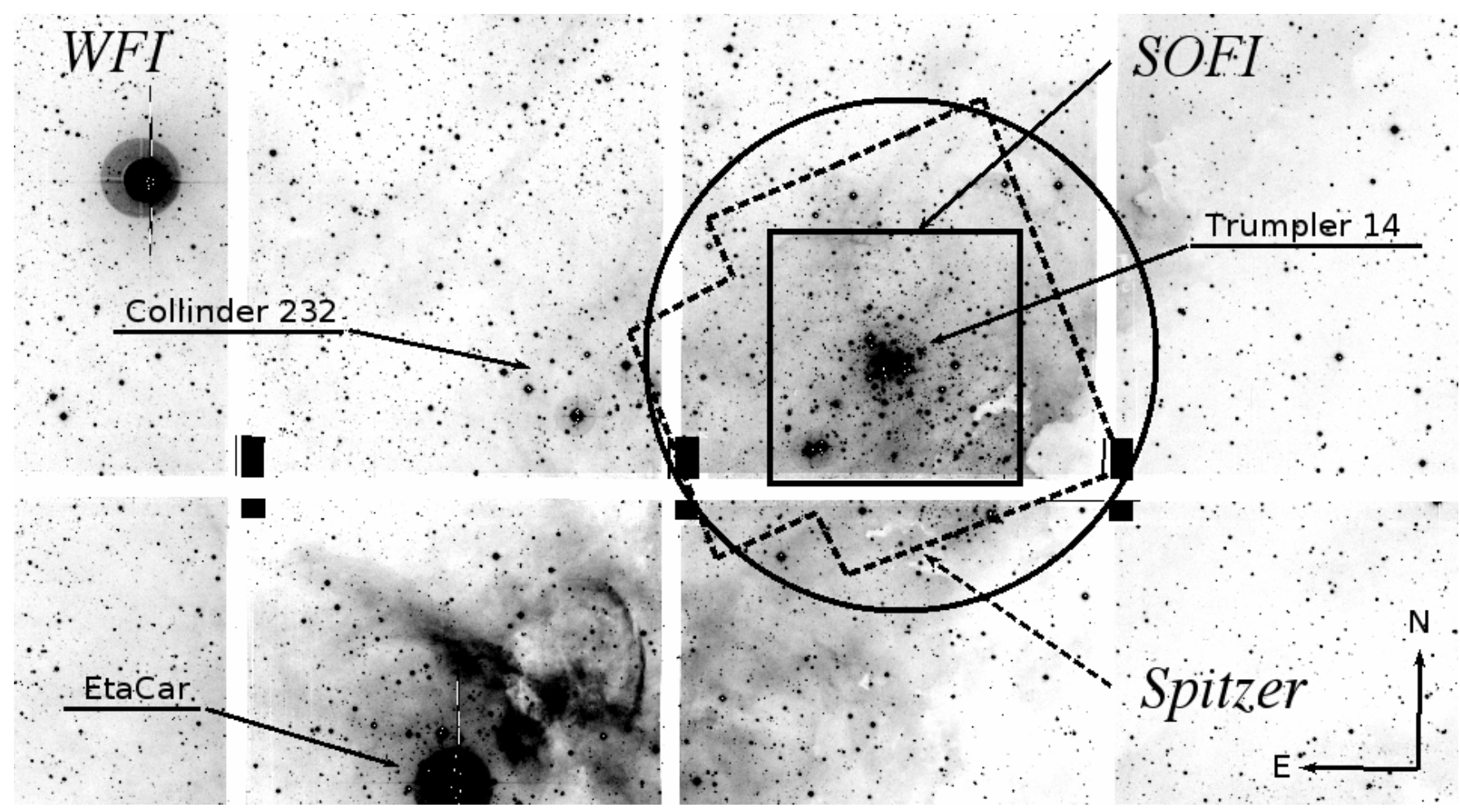

Fig. 1. Zoomed $27^{\prime} \times 15^{\prime}$ mosaic image obtained with the $I$ band filter on the WFI used to sample Trumpler 14 . The circle shows the area of $5^{\prime}$ radius discussed in the text. The dashed and solid polygons show the area covered by the Spitzer and SOFI data, respectively. The location of Trumpler 14 and Collinder 232 are shown together with $\eta$ Carinae. The gaps between the chips of the WFI mosaic are also visible.

(Hur et al. 2012, hereafter H12), whose properties have been thoroughly addressed in the literature. The presence of variable extinction across the cluster, as well as hints of a substantial PMS population were initially reported by Vazquez et al. (1996), and later confirmed by Tapia et al. (2003), Sana et al. (2010), and H12. Tr 14 is relatively close to the Sun, at a distance of $2.5 \mathrm{kpc}$ (Carraro et al. 2004), and therefore is an ideal candidate to study in detail the early stages of stellar evolution and mass accretion.

\section{Observations and data reduction}

In this work we have used panchromatic archival observations from three different facilities, namely i) the Wide Field Imager (WFI) at the MPG/ESO $2.2 \mathrm{~m}$ telescope at La Silla (Chile); ii) the near-infrared (near-IR) SOFI camera at the ESO New Technology Telescope (NTT); and iii) the Infrared Array Camera (IRAC) on the Spitzer Space Telescope. The details of the observations and of their analyses are provided in Sects. 2.1-2.3. In this way we derived three photometric catalogues, all calibrated and homogeneously registered to the same absolute astrometric system. In Fig. 1 we show a schematic view of the combined dataset centred on Tr 14. The study of the PMS objects presented in this paper refers to an area of $\sim 5^{\prime}$ radius around the cluster's centre, which includes the WFI, SOFI, and Spitzer datasets.

Most of the results and discussion in this work concern the physical properties of PMS in stars in Tr 14 as derived from the WFI catalogue at visible wavelengths, but we will also make use of the SOFI and Spitzer near-IR catalogues in Sect. 7, where we discuss the complete spectral energy distribution (SED) of these objects. In a forthcoming paper (Beccari et al., in prep.), we will present a complete study of all PMS stars over the entire field covered by the observations, including those in Trumpler 16 and Collinder 232.

\subsection{WFI observations}

We retrieved from the ESO archives a set of multiband observations made with the WFI on 27 June 2003 (proposal 069.C-0426(C); principal investigator J. Alves). The WFI consists of a mosaic of eight CCD chips with a global field of view $(\mathrm{FoV})$ of $\sim 33^{\prime} \times 34^{\prime}$ and with a pixel size of $0{ }^{\prime} .238$. The Carina Nebula was observed in the $U, B, V, R$, and $I$ bands and through the $\mathrm{H} \alpha$ narrowband filter under good seeing conditions $\left(0.8-1{ }^{\prime \prime} .2\right)$. The details of the observations are listed in Table 1. The observations were made in such a way to place the luminous blue variable $\eta$ Car inside chip No. 56 of the camera array and to include the star clusters $\operatorname{Tr} 14$, Trumpler 16, and Collinder 232 inside the field of view.

We corrected the raw WFI images for bias and flat field, and the overscan region was trimmed using standard IRAF ${ }^{1}$ tools. The photometry was carried out using the standard point spread function (PSF) fitting independently on each image using the DAOPHOTII/ALLSTAR routines (Stetson 1987). A master list was created using the stars detected in at least six of the $V-, R-$, and $I$-band images. This strategy allowed us to remove spurious detections from the final catalogue such as cosmic ray hits or star-like peaks on haloes, and spikes around saturated stars. The master list was used as input for ALLFRAME (Stetson 1994). The average of the single frame's magnitudes for each object in the master list was adopted as stellar magnitude in the final

1 IRAF is distributed by the National Optical Astronomy Observatories, which is operated by the Association of Universities for Research in Astronomy, Inc., under contract to the National Science Foundation 
Table 1. Log of the WFI observations.

\begin{tabular}{lcccc}
\hline \hline Filter & $\begin{array}{c}C W \\
{[\mathrm{~nm}]}\end{array}$ & $\begin{array}{c}F W H M \\
{[\mathrm{~nm}]}\end{array}$ & $N$ & $\begin{array}{c}\text { Exp. time } \\
{[\mathrm{s}]}\end{array}$ \\
\hline U50_ESO877 $(U)$ & 340.4 & 73.2 & 4 & 200 \\
B123_ESO878 $(B)$ & 451.1 & 133.5 & 4 & 200 \\
V89_ESO843 $(V)$ & 539.6 & 89.4 & 4 & 200 \\
RC162_ESO844 $(R)$ & 651.7 & 162.2 & 4 & 200 \\
I203_ESO879 $(I)$ & 826.9 & 203.0 & 4 & 120 \\
H $\alpha 7 \_E S O 856(\mathrm{H} \alpha)$ & 658.8 & 7.4 & 4 & 1200 \\
\hline
\end{tabular}

Notes. Together with the band and filter name, the table lists the central wavelength $(C W)$ and full width at half maximum $(F W H M)$ of the filters, the number of exposures and their total duration.

catalogue, while we used the standard deviation as associated photometric uncertainty. We looked for variable stars in the final photometric catalogue, but no systematic variability within the photometric uncertainties was observed between these relatively short exposures.

The instrumental positions, in pixels, were transformed into J2000 celestial coordinates by means of an astrometric solution (in the form of a second degree polynomial) obtained with CataXcorr ${ }^{2}$ from more than 30000 stars in common between our final WFI catalogue and the 2MASS catalogue of the same region. The rms scatter of the solution is less than 0.' 3 in both right ascension (RA) and declination (Dec).

The calibration of the instrumental magnitudes was done following the procedure described in Da Rio et al. (2009, hereafter D09). As shown in that work, the transformations between the WFI instrumental photometric system and the standard JohnsonCousins system are non-linear, especially when a wide colour (i.e. temperature) range is considered. We thus decided not to apply any colour correction to our instrumental magnitudes, and to keep them in the WFI photometric system as defined by the throughput of the adopted WFI filters. Given the instrumental magnitude in a given band $m_{\mathrm{WFI}}^{\text {ins }}$, corrected for the air mass of the science observations, the absolute photometric calibration in the WFI-VegaMag photometric system will be derived as $m_{\mathrm{WFI}}^{\text {vega }}=m_{\mathrm{WFI}}^{\text {ins }}+Z \mathrm{p}$, where $Z \mathrm{p}$ is the photometric zero point. In practice, $Z \mathrm{p}$ is the value one would obtain for a Vega-like star when all the colours are equal to zero (see D09 for details).

We derived the value of $Z p$ for each band by using a set of photometric standards acquired during the night of the observations. Since the $\mathrm{Zp}$ values that we calculated are identical, within the uncertainties, to those derived in 2003 March by the WFI support team on a photometric night and published by the observatory ${ }^{3}$, we used the latter to calibrate our data. Since the $Z$ p value is not available in that list for the $\mathrm{H} \alpha$ band, we derived it directly with the procedure indicated above, obtaining $\mathrm{Zp}(\mathrm{H} \alpha)=21.630 \pm 0.096$.

\subsection{SOFI observations}

We used a set of near-IR observations made with the SOFI camera in large field mode on the night of 2 May 2008 (proposal 078.D-0371(A); principal investigator C. Weidner). In this mode the camera has a pixel scale of 0 '.288, allowing us to sample a region of $4^{\prime} .9 \times 4^{\prime} .9$ around the centre of the cluster (see Fig. 1).

\footnotetext{
2 The software is available for download at http://davide2.bo. astro.it/ paolo/Main/CataPack.html

3 http://wWw.eso.org/sci/facilities/lasilla/

instruments/wfi/inst/zeropoints/
}

Exposures through the $J$ and $K$ filters were obtained with slightly different detector setups, namely DIT $=4 \mathrm{~s}$ and NDIT $=10$ for the $J$ band and DIT $=4 \mathrm{~s}$ and NDIT $=20$ in $K$. The same exposure was repeated 20 times in $J$ and 40 times in $K$ with a random dithering pattern for background correction purposes. During the observations the average seeing was $\sim 1^{\prime \prime}$.

Images were pre-reduced using standard IRAF routines. A sky image, obtained by median combination of the dithered frames of each filter, was subtracted from each frame. Flat fielding was then done using the SpecialDomeFlat template, which applies the appropriate illumination corrections, as described in the SOFI User Manual (Lidman \& Cuby 2000). Finally, for each filter all the dithered frames obtained in a sequence were aligned in coordinates and then averaged in a single image. Standard crowded field photometry, including PSF modelling, was then carried out on each of the averaged frames using DAOPHOTII/ALLSTAR.

We used hundreds of stars in common with the 2MASS catalogue in order to obtain an absolute astrometric solution for the stars in the SOFI catalogue. The same stars were also used as secondary photometric standards in order to calibrate the SOFI $J$ and $K$ magnitudes with the 2 MASS photometry.

\subsection{Spitzer observations}

Finally, we retrieved from the Spitzer Enhanced Imaging Products archive ${ }^{4}$ two near-IR images (at $3.6 \mu \mathrm{m}$ and $4.5 \mu \mathrm{m})$ centred on $\operatorname{Tr} 14$, obtained with the IRAC camera (proposal 30734; principal investigator D. Figer). The two mosaic images are the combination of a number of sub-images and have a pixel scale of 0.'6. Standard PSF photometry was performed by modelling the PSF on each image, producing a catalogue listing the relative positions and magnitudes of stars in common between the two bands.

The calibration of the Spitzer observation was done following the IRAC instrument handbook ${ }^{5}$ (see also Hora et al. 2008). We performed aperture photometry with the IRAF/DAOPHOT task on more than 40 well sampled and isolated stars in each frame using an aperture of 4 pixels while the background was locally sampled in a annulus between 4 and 12 pixels, following the IRAC instrument handbook. We used these stars as secondary photometric standards to calibrate the final photometric catalogue. Moreover, several hundred stars in common with the SOFI catalogue were used to extend the astrometric solution to the Spitzer catalogue.

\section{Differential reddening}

Since the stars in our sample are still embedded in the molecular cloud, a large amount of differential reddening is expected to affect the photometry. Recently H12 carried out a detailed study of the differential reddening in the same region, finding that inside the cluster the extinction law is characterized by $R_{V}^{\mathrm{cl}}=4.4 \pm 0.2$. They also determined the foreground reddening to be $E(B-V)_{\mathrm{fg}}=0.36 \pm 0.04$ for the stars in the $\eta$ Carinae nebula, based on the properties of 141 early-type stars with high membership probability from proper motion studies. They derived a distance modulus of $12.3 \pm 0.2$, corresponding to a distance of $2.9 \pm 0.3 \mathrm{kpc}$ for $\operatorname{Tr} 14$ and $\operatorname{Tr} 16$ and concluded that the two clusters are at the same distance. Finally, they derived

\footnotetext{
4 http://irsa.ipac.caltech.edu/data/SPITZER/Enhanced/ Imaging/index.html

5 http://irsa.ipac. caltech.edu/data/SPITZER/docs/i
} 


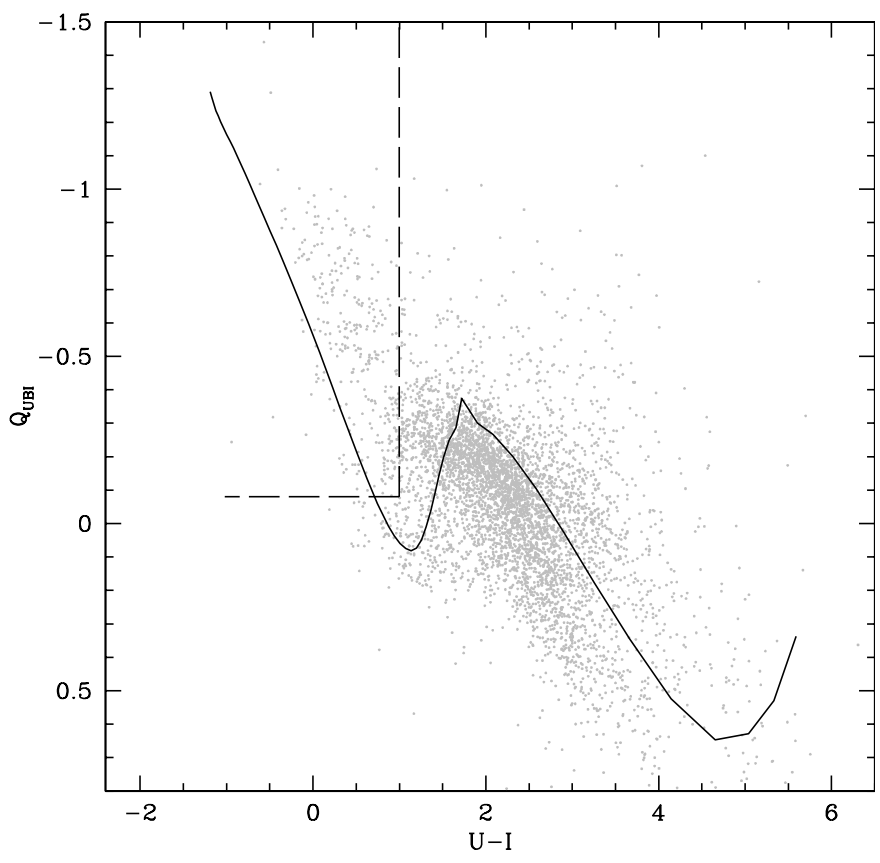

Fig. 2. Solid line: reddening free colour $Q_{U B I}$ as a function of $U-I$ as derived from the model atmospheres of Bessell et al. (1998) and reddened by the foreground extinction (see details in the text), whereas grey dots: stars in our catalogue. Only stars with $U-I<1$ and $Q_{U B I}<-0.1$ (area inside the dashed lines) were used to generate our reddening map.

a reddening map (see their Fig. 6) using the early-type stars in the region, and used it to correct the magnitudes of the entire population, including those of PMS stars.

Taking advantage of the large number of filters available in our photometry, we decided to independently derive a reddening map using the method described in Mihalas \& Binney (1981) and Romaniello et al. (2002, hereafter R02). We adopted the reddening free colour $Q_{U B I}$ defined as follows:

$Q_{U B I}=(U-B)-\frac{E(U-B)}{E(B-I)}(B-I)$.

The reddening-free colour $Q_{U B I}$ computed for the observed stars (grey points) and for the atmospheric model of Bessell et al. (1998, solid line) is shown in Fig. 2 as a function of $(U-I)$. The theoretical model shown in the figure is already reddened by the foreground extinction as found by $\mathrm{H} 12$.

The effect of extinction is to move the stars horizontally from the zero-reddening locus, i.e. the location of the model in the colour-colour diagram. We can simply derive information on the interstellar reddening by comparing the colours of the observed stars with those of the models, in the assumption that the displacement of the stars from the $\mathrm{W}$-shaped model is only induced by the differential extinction.

As shown in Fig. 2, only the stars with $U-I<1$ and $Q_{U B I}<-0.1$ show a unique solution. Moreover, it's important to stress that the stars with $Q_{U B I}<-0.5$ and $U-I>1$ cannot be safely used to study the reddening map because this region of the diagram is mostly populated by PMS stars whose colours are clearly affected by $U$-band excess. These limits define a selection box shown with the dashed lines in the figure. In order to build a reddening map for the entire region covered by our WFI observations we first derived the value of visual extinction $A_{V}=R_{V} \times E(B-V)$ for the stars in the selection box. While, as previously explained, $E(B-V)$ can be
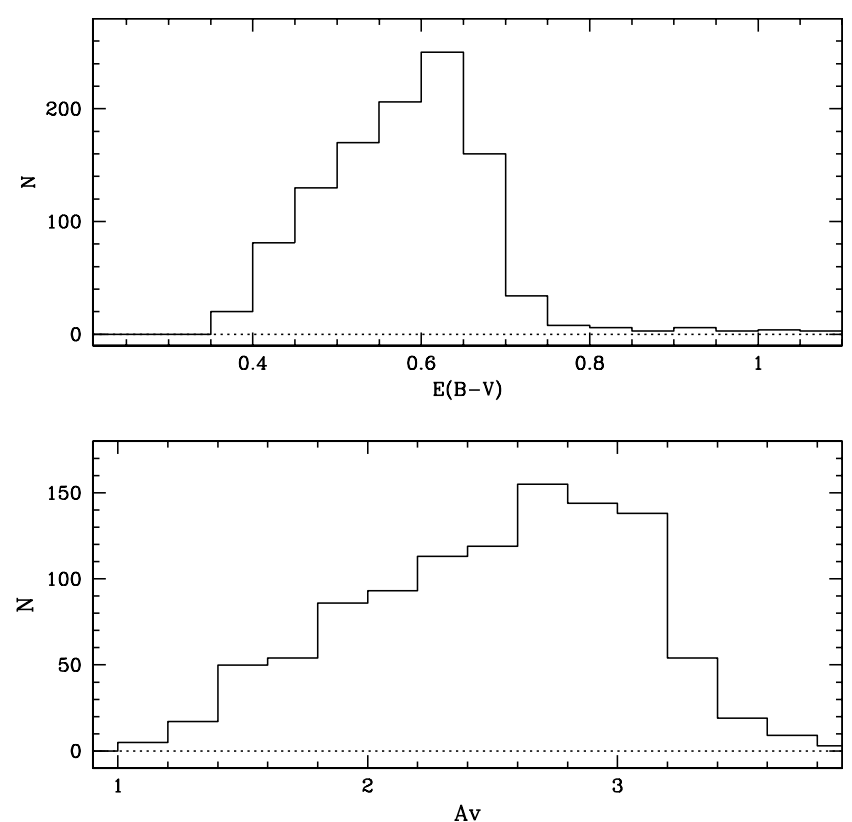

Fig. 3. $E(B-V)$ (upper panel) and Av (lower panel) distribution as derived from the reddening free colours in the region sampled by the WFI data.

derived by horizontally projecting the stars to the model in the $Q_{U B I}$ vs. $U-I$ plane of Fig. 2, we need to assume an extinction law $R_{V}$ in order to derive $A_{V}$. Following the recent results from $\mathrm{H} 12$ we estimated the total $A_{V}$ of the selected stars as $A_{V}=3.1 \times E(B-V)_{\mathrm{fg}}+R_{V, \mathrm{cl}} \times\left(E(B-V)-E(B-V)_{\mathrm{fg}}\right)$, where the only parameter missing is $E(B-V)$ which we can estimate using the $Q_{U B I}$ parameter.

In Fig. 3 we show the histograms of the values of the total extinction $A_{V}$ (lower panel) and selective extinction $E(B-V)$ (upper panel) as calculated using more than 1000 stars located within the selection box of Fig. 2. The median of the $E(B-V)$ distribution is $0.59 \pm 0.06$ with a minimum value of 0.075 and a maximum value of 1.425 in agreement with the findings of H12 and of Carraro et al. (2004). In order to produce an extinction map, the entire FoV was divided into a grid of $3^{\prime} \times 3^{\prime}$ cells, for a total of 64 cells. The extinction in each of them was obtained as the average value of the extinction of the individual blue stars in that cell and this value was assumed as representative for all stars falling inside that cell. The typical standard deviation of the mean of $E(B-V)$ of the individual blue stars in the cells in the area of $5^{\prime}$ around the centre of trumpler 14 (the region studied in detail in this paper) is $\sim 0.08$, which translates into an uncertainty of $\sim 0.35$ on $A_{V}$ because $R_{V}=4.4$. We used this map to correct all the available magnitudes for extinction, from the optical through to the IR, following the extinction law of H12. As we will show later and as already pointed out by H12, the stars in the foreground will appear too blue when corrected with this map and will need to be treated separately.

\section{The colour-magnitude diagram}

The optical colour-magnitude diagram (CMD) of the inner $5^{\prime}$ of Tr 14 is shown in Fig. 4. Stars highlighted with large dot are bona fide PMS. The selection criteria and physical parameters for this class of object will be discussed in detail in this section and in Sect. 5, respectively.

All magnitudes are corrected for extinction on the basis of our extinction map. Typical photometric uncertainties (on magnitudes and colours) are shown by the crosses for various 
G. Beccari et al.: Mass accretion rates from multiband photometry in the Carina Nebula: the case of Trumpler 14

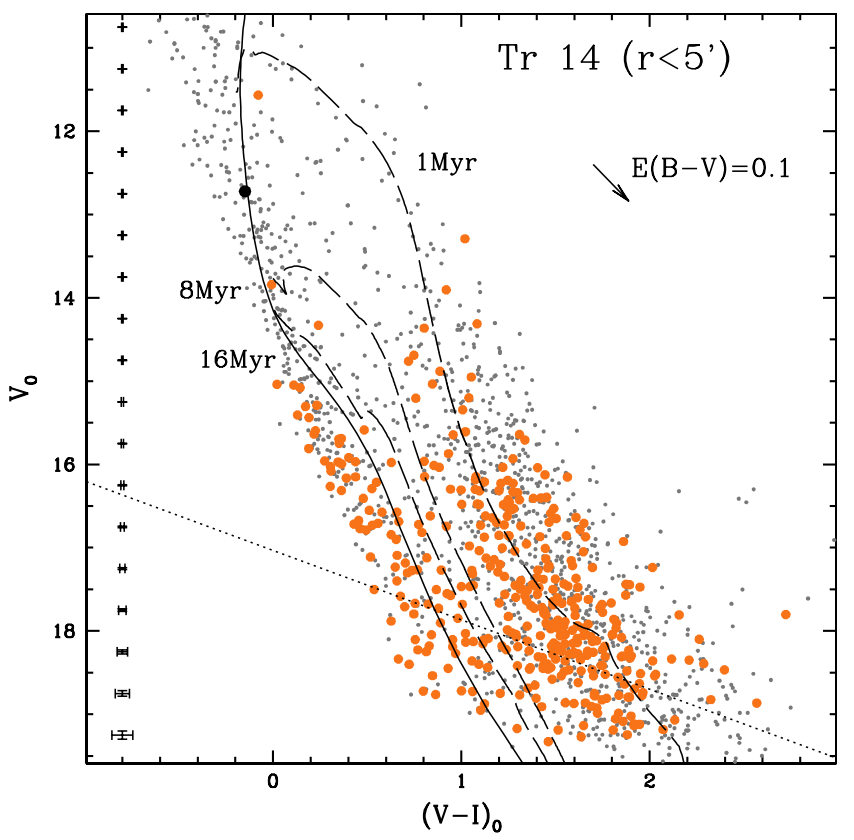

Fig. 4. CMD of all stars within $5^{\prime}$ of the cluster centre, after correction for extinction. Stars highlighted with large dots (orange in the electronic version) are bona fide PMS. The selection of there stars and the physical parameters are discussed later in the paper. Three PMS isochrone (Degl'Innocenti et al. 2008) for ages of 1,8 and $16 \mathrm{Myr}$ are shown as reference. The ZAMS from Marigo et al. (2008) for solar metallicity and the assumed distance modulus $(m-M)_{0}=12.3$ is shown by the solid line. The typical uncertainties on magnitudes and colours are indicated by the crosses. The dotted line shows the $50 \%$ completeness limit of the photometry. A reddening vector proportional to $E(B-V)=0.1$ (i.e. the typical uncertainty) is also shown.

magnitudes. In the figure we also show the position of the zero age main sequence (ZAMS), taken from the models of Marigo et al. (2008) for solar metallicity (solid line). We have adopted as distance modulus the value given by H12, namely $(m-M)_{0}=$ 12.3. The position of the ZAMS obtained in this way helps us to identify a population of candidate MS stars that extend from the saturation limit at $V_{0} \simeq 11$ down to $V_{0} \simeq 19$.

In order to measure the completeness of our photometry, we made extensive use of artificial stars experiments, following the recipe described in Bellazzini et al. (2002). More than 300000 artificial stars were uniformly distributed on the WFI chips in groups of 4000 stars at a time in order to avoid changing the crowding conditions. We produced a catalogue of simulated stars with a $V$ magnitude, $V-I$ and $V-\mathrm{H} \alpha$ colours randomly extracted from a luminosity function, and colour distributions modelled to reproduce the observed distribution.

The whole data reduction process was repeated as for the actual observations, and the fraction of recovered stars was estimated at each magnitude level. The limit of $50 \%$ of photometric completeness within $5^{\prime}$ of the centre of $\operatorname{Tr} 14$ is shown by the dotted line in Fig. 4. From a comparison with the theoretical model we estimate that we sample the PMS stars down to $0.7 M_{\odot}$ and $1 M_{\odot}$ with a $50 \%$ photometric completeness, with the lower masses sampled at the younger ages.

A discontinuity in the stellar colour distribution in this CMD separates a population of objects along the ZAMS from a population clearly grouped at redder colours and consistent with the young population of PMS stars already detected in $\operatorname{Tr} 14$ (H12; Ascenso et al. 2007). This confirms that Tr 14 is an active starforming region. In order to learn more about the properties of

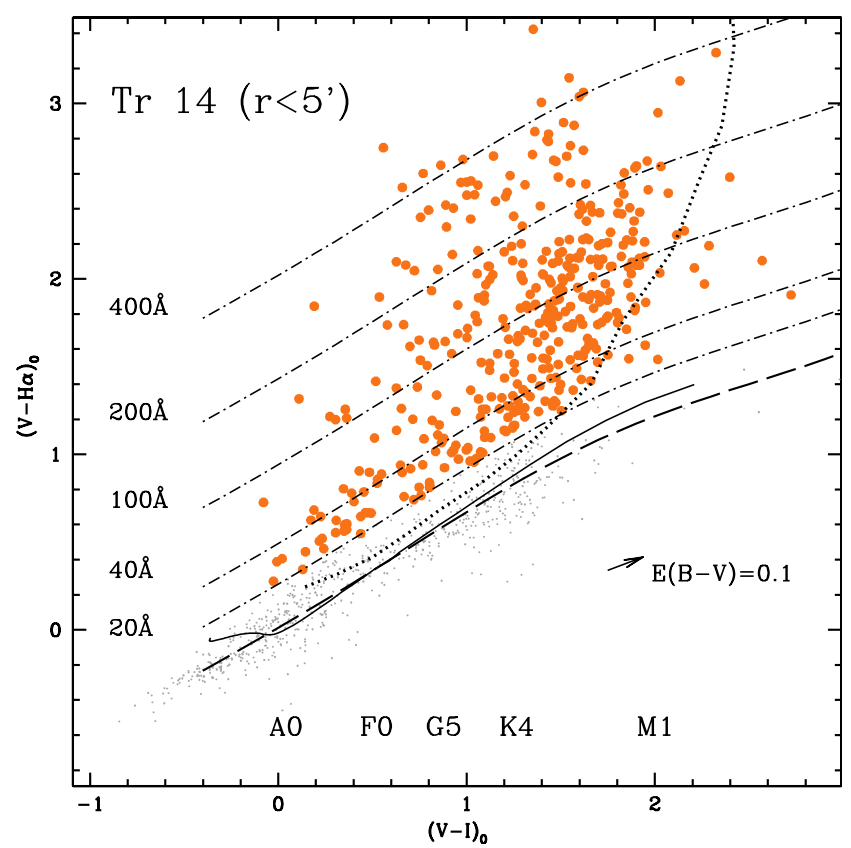

Fig. 5. Colour-colour diagram of all stars after correction for reddening. Stars in light grey correspond to objects sampled in the first $5^{\prime}$ from the cluster centre. The solid line represents the median $(V-\mathrm{H} \alpha)_{0}$ colour of stars with an error on $(V-\mathrm{H} \alpha)_{0}<0.05$, and is defined as the locus of stars without $\mathrm{H} \alpha$ excess emission and hence the location of stars with $E W(\mathrm{H} \alpha)=0$. Objects indicated as large dots (orange in the electronic version) correspond to stars with $\mathrm{H} \alpha$ excess emission. The solid line shows the location on the CMD of atmospheric models of Bessell et al. (1998). The model is in excellent agreement with the observations. The dotted line marks the 50\% completeness limit of the photometry. Spectral types are also indicated. A reddening vector proportional to $E(B-V)=0.1$ (i.e. the typical uncertainty) is also shown.

this recent star formation episode, we followed the method developed by DM10 to identify bona fide PMS stars. A population of candidate PMS stars is clearly visible at $V-I \gtrsim 1$, well separated from the population of MS stars. These PMS stars have been recently studied by H12, who selected them based on their position in the optical CMD. In this work, we apply the method developed by DM10 to look for the signature of the active mass accretion process that is expected to characterize the PMS phase and that is responsible for the strong $\mathrm{H} \alpha$ excess emission normally observed in objects of this type (e.g. Calvet et al. 2000).

In Fig. 5 we show the distribution of the stars in the $V-I$ vs. $V-\mathrm{H} \alpha$ colour-colour diagram, corrected for extinction. We use the median $(V-\mathrm{H} \alpha)^{\text {ref }}$ de-reddened colour of stars with small $(<0.05 \mathrm{mag})$ combined photometric uncertainty in the $V, I$, and $\mathrm{H} \alpha$ bands (grey dots in Fig. 5), as a function of $V-I$, to define the reference template with respect to which the excess $\mathrm{H} \alpha$ emission is identified (dashed line).

We selected a first sample of stars with excess $\mathrm{H} \alpha$ emission by considering all those with a $\Delta(\mathrm{H} \alpha)=(V-\mathrm{H} \alpha)^{\mathrm{star}}-(V-$ $\mathrm{H} \alpha)^{\text {ref }}$ at least four times larger than the photometric uncertainty on the $(V-\mathrm{H} \alpha)^{\text {star }}$ colour. Then we calculated the EW of the $\mathrm{H} \alpha$ emission line, $E W(\mathrm{H} \alpha)$, from the measured colour excess using Eq. (4) of D10. We finally considered as bona fide PMS stars those objects with $E W(\mathrm{H} \alpha)>20 \AA$ (see D10 and references therein). This allows us to safely remove possible contaminants from our sample, such as older stars with chromospheric activity and Ae/Be stars (see White \& Basri 2003; Beccari et al. 2014).

We visually inspected the position of all the candidate $\mathrm{H} \alpha$ emitters on the $V, I$, and $\mathrm{H} \alpha$ images and excluded from the 


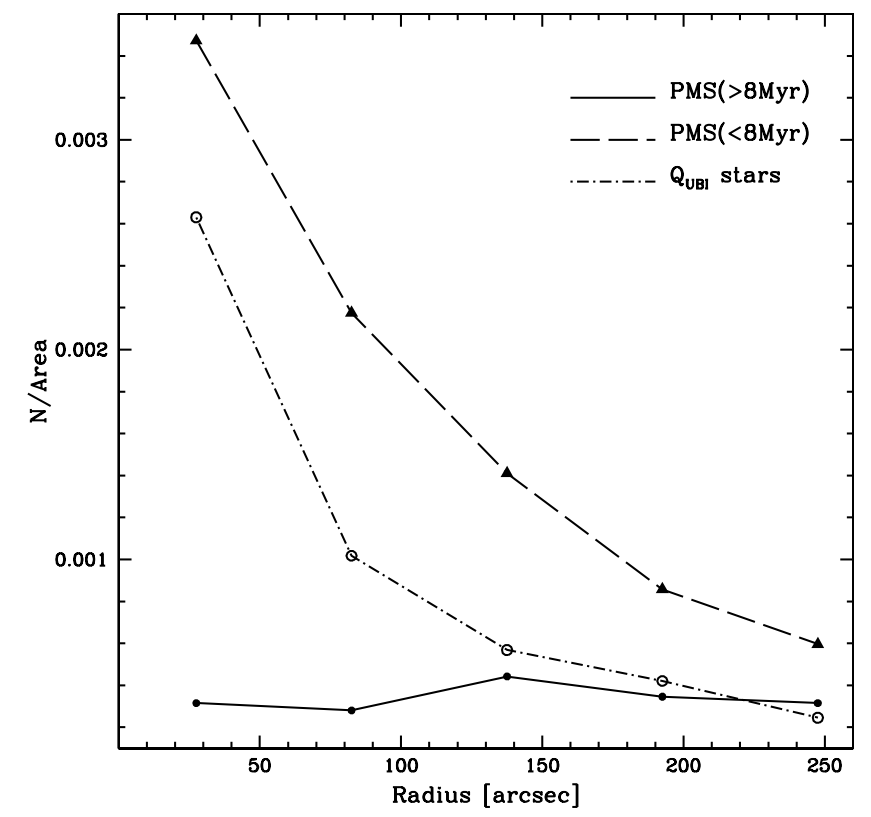

Fig. 6. Radial density distribution of old (age $>8 \mathrm{Myr}$ ) and young (age $<8 \mathrm{Myr}$ ) bona fide PMS together with the stars used in Sect. 3 to calculate the reddening map of the region.

final list all those stars falling on a filamentary structure of the cloud in which the cluster is embedded. Even if these stars could in principle be genuine $\mathrm{H} \alpha$ excess sources (and hence considered PMS stars), the fact that the scale of the background spatial variation is equal to or smaller than the full width at half maximum of the PSF makes the background determination too prone to errors. As a consequence, the magnitude of these stars are too uncertain and we do not consider them as bona fide PMS objects. After this conservative selection, we count a total of 389 PMS stars in our sample, with a median $E W(\mathrm{H} \alpha)=90 \AA$. Hereafter we will refer to these objects as bona fide PMS stars.

The positions of these stars in the optical CMD is shown in Fig. 4 together with a ZAMS from Marigo et al. (2008, solid line) and three PMS isochrone for ages of 1, 8, and 16 Myr from Degl'Innocenti et al. (2008, dashed lines) for solar metallicity.

\subsection{The reddening correction of the old PMS stellar population}

Using the same approach as described by De Marchi et al. (2011b), we perform a preliminary study of the radial distribution of the candidate PMS stars at different ages. In particular, using the $8 \mathrm{Myr}$ isochrone as references, we divided the population of bona fide PMS into an older ( $>8 \mathrm{Myr}$ ) and younger ( $<8 \mathrm{Myr}$ ) subgroups. In Fig. 6 we show the radial density distribution of the two subgroups of PMS objects, with respect to the centre of $\operatorname{Tr} 14$. The solid and dashed lines correspond, respectively, to the older and younger populations, while the distribution of upper main-sequence (MS) stars used in Sect. 3 to derive the reddening map of the region is shown by the dot-dashed line. The figure clearly shows that objects with $\mathrm{H} \alpha$ excess older than $\sim 8$ Myr have a different radial distribution from that of the cluster's stars used to calculate the reddening map.

The implication of the differences in the radial distribution of the PMS populations will be discussed in greater detail in Sect. 5. For the moment, Fig. 6 implies that we can safely use the reddening map to correct the magnitudes of the younger bona fide PMS objects for differential reddening, by assuming that the placement of these PMS objects with respect to the cluster's absorbing material is similar to that of the reference stars in its vicinity. This assumption is clearly not valid for the older $\mathrm{H} \alpha$ excess emitters. We also note that the older PMS stars are not in regions of particularly high reddening, confirming that they should have lower extinction than younger PMS stars. At the same time, as explained in Sect. 3, the typical uncertainty on $E(B-V)$ is $\sim 0.1$. It is then clear that the reddening uncertainty has a marginal effect on the distribution of the stars in the diagrams.

In order to obtain a more realistic estimate of the reddening of the older population of PMS, we can use the stars with $\mathrm{H} \alpha$ excess that appear bluer than the ZAMS (e.g. Fig. 4). By definition the ZAMS represents the theoretical location of the stars at the beginning of the hydrogen burning phase in the core, i.e. the MS stage, or conversely, the end of the PMS phase. Observationally, the ZAMS represents the bluest end of the colour distribution of the PMS population in a CMD. We then decided to correct the magnitudes of the older population of PMS stars using a constant value of $A_{V}=1.7$, which ensures that the ZAMS constrains the blue envelope to at least $80 \%$ of the old PMS objects at $V_{0} \simeq 16-18$ in Fig. 4, i.e. to the stars in this subgroup with the smallest uncertainty on magnitude and colour. For the faintest stars, the increase of the photometric uncertainties can easily be at the origin of some residual colour scatter around the ZAMS. While the choice of $80 \%$ is somewhat arbitrary, it is important to stress here that changing this value to $50 \%$ or $100 \%$ would not change any of the results of the paper.

Finally, we revised the selection of the $\mathrm{H} \alpha$ emitters (bona fide PMS stars), In fact, even if the reddening vector runs almost parallel to the median photospheric colours of normal stars in the $V-\mathrm{H} \alpha$ vs. $V-I$ colour-colour diagram (see De Marchi et al. 2011b), we calculated the new values of $E W(\mathrm{H} \alpha)$ after the application of the new reddening correction to the $V, I$ and $\mathrm{H} \alpha$ magnitudes of the old PMS objects. We find that 33 stars now show $E W(\mathrm{H} \alpha)<20 \AA$ and, following our stringent selection criteria reported above, these stars cannot be classified as $\mathrm{H} \alpha$ excess emitters and will not be considered in this work. The population of bona fide PMS stars in Tr 14 finally includes 356 objects.

\section{Physical parameters of the PMS stars}

The luminosities $(L)$ and effective temperatures $\left(T_{\text {eff }}\right)$ of all stars within $5^{\prime}$ of the cluster centre are shown in the H-R diagram (Fig. 8). PMS stars are shown as thick dots (orange in the electronic version), whereas all other objects are in grey.

Effective temperatures $T_{\text {eff }}$ were determined by fitting the de-reddened $(V-I)_{0}$ colours of the stars with the ones computed for the same WFI bands using stellar atmosphere models. We adopted the model atmospheres of Bessell et al. (1998), which provide an excellent fit to our data (solid line in Fig. 5). The de-reddened magnitudes were then compared with the absolute magnitudes of the models in order to derive the stellar radius. The bolometric luminosity was finally derived, adopting the distance modulus $(m-M)_{0}=12.3 \pm 0.2$ from $\mathrm{H} 12$ and by comparing the stellar radius and $T_{\text {eff }}$ with those of the Sun (see Romaniello et al. 2002, for further details).

We also show in Fig. 8 the evolutionary tracks for masses of $0.6,0.8,1,1.2,1.4,1.6,2$, and $3 M_{\odot}$ (dashed lines) and isochrones for ages of $0.25,0.5,1,2,4,8,16$, and $32 \mathrm{Myr}$ (dotted lines), from the PMS evolutionary models of the Pisa group (Degl'Innocenti et al. 2008; Tognelli et al. 2012). The 
G. Beccari et al.: Mass accretion rates from multiband photometry in the Carina Nebula: the case of Trumpler 14

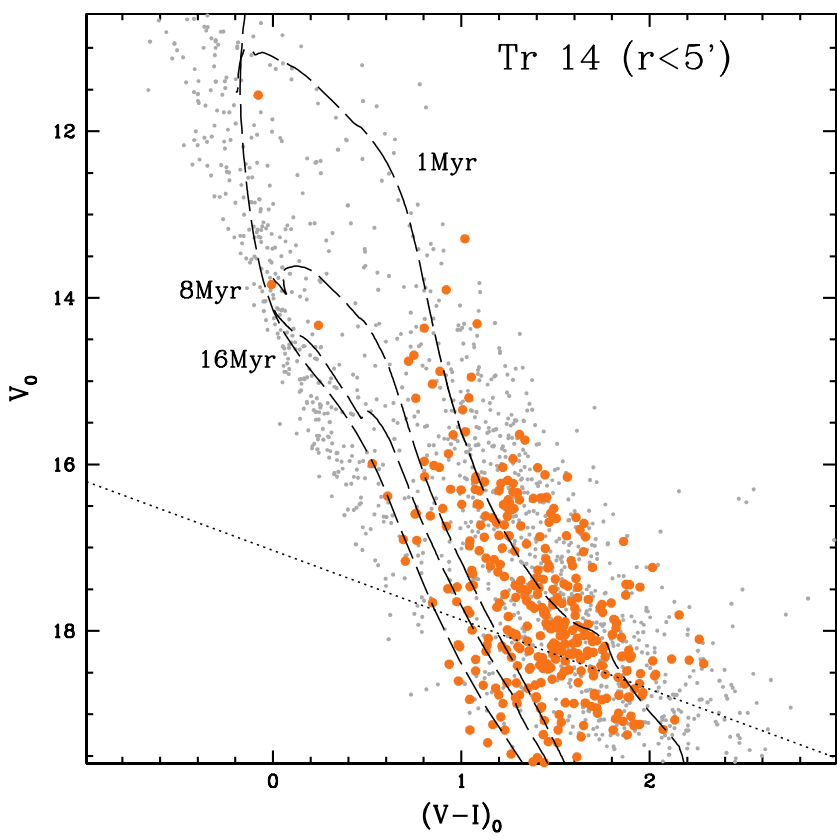

Fig. 7. CMD of all stars within $5^{\prime}$ of the cluster centre, after correction for extinction. The symbol types are the same as used in Fig. 4. A reddening correction with a constant $A_{V}=1.7$ has been used for the PMS stars older than $8 \mathrm{Myr}$ in Fig. 4. The dotted line marks the 50\% completeness limit of the photometry.

models were calculated for $Z=0.014, Y=0.2533$ and a mixinglength parameter $\alpha=1.68$, applicable to Tr 14. In Fig. 8 we explicitly label the tracks for 1,2 , and $3 M_{\odot}$ and the location of the $4 \mathrm{Myr}$ isochrone, for reference.

In order to study in greater detail the physical properties of the 356 PMS in Tr14, we used a finer grid of models than the one shown in Fig. 8 to derive a reliable measure of the mass and age (see Romaniello et al. 1998, for details on the method used, with an approach similar to the one more recently presented by Da Rio et al. 2012). Mass and age distributions for the PMS objects are shown in the upper and lower panels of Fig. 9, respectively.

The mass distribution peaks around $1 M_{\odot}$. As expected, the number of stars with masses above $\sim 1 M_{\odot}$ decreases, because the timescales of PMS evolution are smaller for more massive objects. On the other hand, the decrease in the number of stars for masses below $1 M_{\odot}$ is due to the drop in the photometric completeness (see the dotted line in Fig. 7).

The median of the age distribution is $\sim 2.8 \mathrm{Myr}$, with an upper quartile of $\sim 6 \mathrm{Myr}$ and a lower quartile of $\sim 1.2 \mathrm{Myr}$. As already noted by Ascenso et al. (2007), it appears that star formation has been ongoing in $\operatorname{Tr} 14$ for at least 5-6 Myr. The available observations do not allow us to conclude whether the star formation in the last 6 Myr was continuous or whether this young population of PMS objects is indeed the outcome of a few major bursts, as postulated by Ascenso et al. (2007).

On the other hand, it is clear that multiple bursts have occurred in this region. The distribution of PMS ages confirms that $\sim 20 \%$ of the objects have ages older than $8 \mathrm{Myr}$, with a median age of $\sim 20$ Myr. This implies that in this region star formation started more than $8 \mathrm{Myr}$ ago, at least as far back as $\sim 20 \mathrm{Myr}$ ago, followed by a major episode in the last 2-6 Myr. A similar behaviour has already been observed in Galactic star forming regions (NGC 3603, Beccari et al. 2010; NGC 6823, Riaz et al. 2012; NGC 6611, De Marchi et al. 2013b) and in the Magellanic

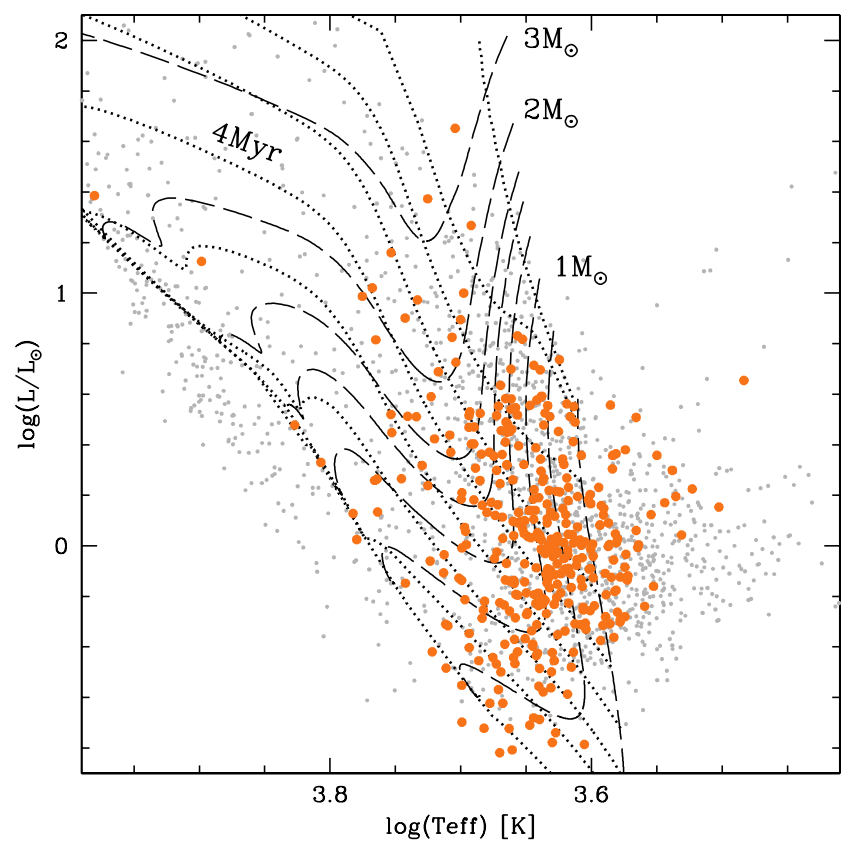

Fig. 8. All objects located within $5^{\prime}$ of the cluster centre are shown in grey in the H-R diagram. Bona fide PMS stars are shown as large dots (orange in the electronic version). Dashed lines show the evolutionary tracks for solar metallicity and masses of $0.6,0.8,1,1.2,1.4,1.6,2$, and $3 M_{\odot}$. The corresponding isochrones are shown as dotted lines, for ages of $0.25,0.5,1,2,4,8,16$, and $32 \mathrm{Myr}$, from right to left. The constant logarithmic age step has been selected in such a way that the typical distance between isochrones is larger than the photometric uncertainties.

Clouds (Panagia et al. 2000; De Marchi et al. 2010, 2011a,b, 2013a; Spezzi et al. 2012).

Our age resolution does not allow us to say whether there were multiple episodes in $\operatorname{Tr} 14$ before the most recent one, nor whether they were as prominent as the one responsible for the 2-4 Myr old population. However, if the fraction of PMS stars with $\mathrm{H} \alpha$ excess has an exponential decay with time (Fedele et al. 2010), episodes occurring more than $10 \mathrm{Myr}$ ago might have contributed a large portion of the stars in this region.

It is important to note that there are a number of uncertainties when searching for age spreads in starburst clusters (see e.g. the reviews by Preibisch 2012; and Soderblom et al. 2013). From a theoretical point of view, while the absolute ages of PMS stars are only as good as the isochrones allow, relative ages are always better defined than a factor of two for ages up to $\sim 30 \mathrm{Myr}$, as already shown by Romaniello et al. (2002) and Da Rio et al. (2010). From an observational point of view, the colours and magnitudes of the stars are affected by uncertainties induced by inaccurate evaluation of differential extinction, stellar variability (Herbst et al. 2002) and complex physical processes like episodic accretion (Baraffe et al. 2009). We stress once again here that the typical uncertainty on $E(B-V)$ in the studied region is of the order of $0.08 \mathrm{mag}$, i.e. $\sim 0.35$ on $A_{V}$. Hence, while an inaccurate estimate of $A_{V}$ would have an impact on the age determination of individual stars, we can exclude that it would have a significant impact in the age spread detected in the region. Unresolved binaries can also affect the apparent distribution of stars in the CMD and lead to an incorrect estimation of the age of such photometrically unresolved systems. We stress that the contamination for stars appearing to be older than $10 \mathrm{Myr}$ should not be more than about 5\% (for 100\% unresolved binaries; Weidner et al. 2009). 

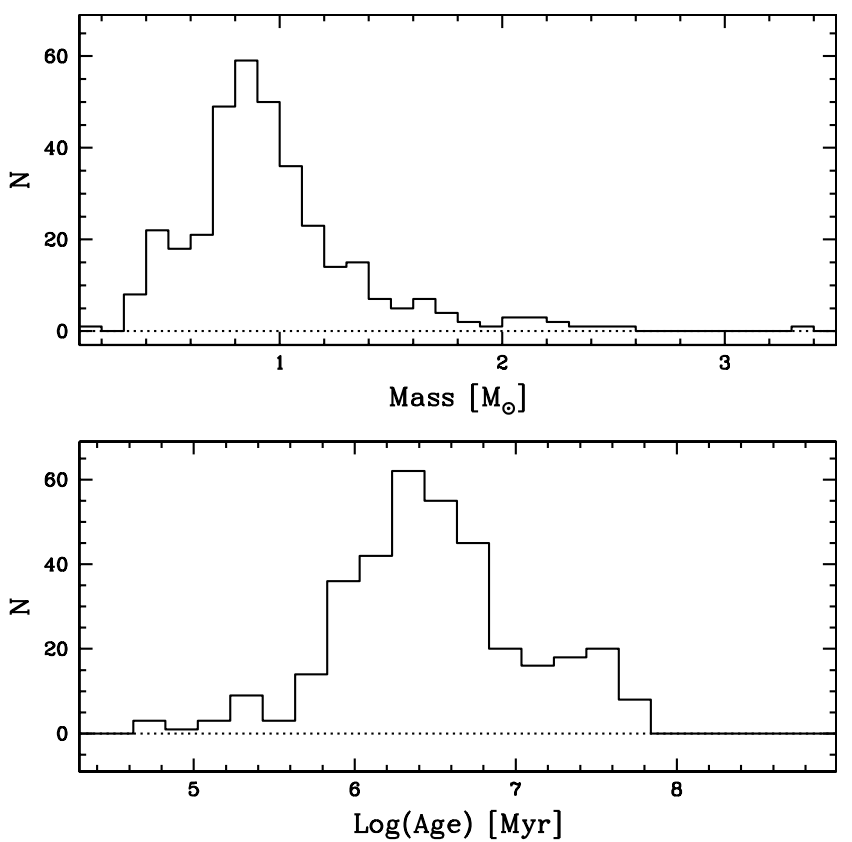

Fig. 9. Mass and age distribution of the bona fide PMS stars (upper and lower panel, respectively).

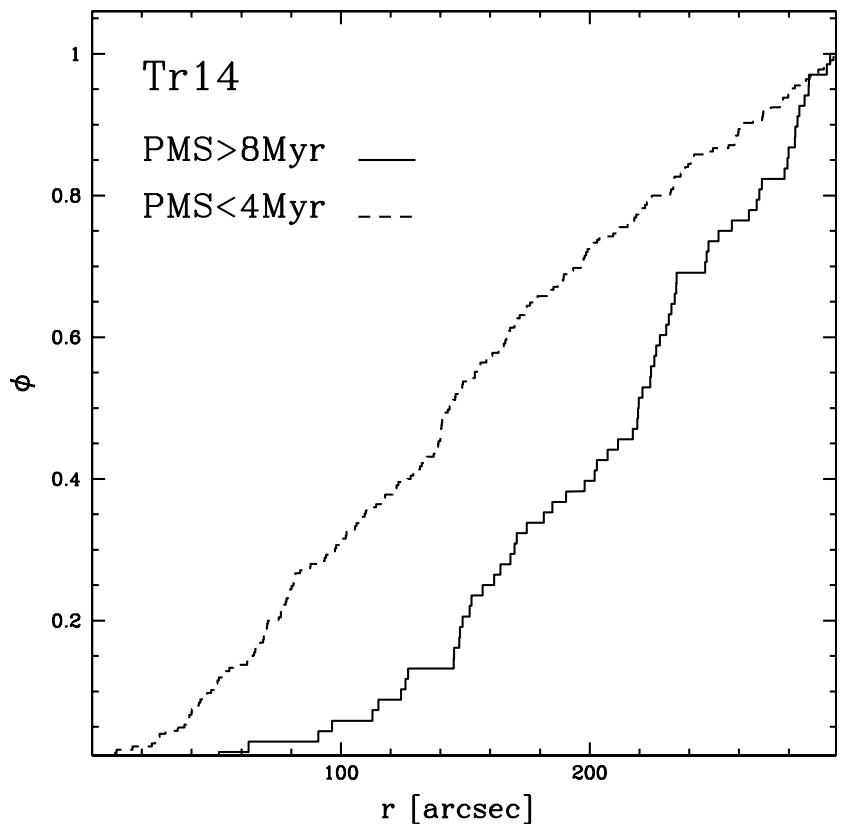

Fig. 10. Cumulative radial distribution of the younger ( $<4 \mathrm{Myr}$; dashed line) and older ( $>8$ Myr; solid line) PMS objects in Tr 14 .

However, none of these effects can explain the different radial distributions of younger and older PMS stars as shown in a number of star-burst clusters (e.g. Beccari et al. 2010; De Marchi et al. 2011a,b, 2013a,b).

Figure 6 already showed that this is the case for $\operatorname{Tr} 14$. To characterize this result in an even more solid statistical sense, in Fig. 10 we compare the radial distribution of the older PMS stars (>8 Myr, solid line) with that of the PMS stars younger than $4 \mathrm{Myr}$ (dashed line), with respect to the cluster centre. The latter age was selected to ensure that most of these stars have the same age as the massive objects that define the cluster, i.e. the $\mathrm{O}$ and B stars.
The figure clearly shows that the younger PMS stars are more centrally concentrated than the older generation, in agreement with the results found e.g. in NGC 3603 and NGC 6611 in the Galaxy (Beccari et al. 2010; De Marchi et al. 2013b) and in 30 Doradus, NGC 346, and NGC 602 in the Magellanic Clouds (see De Marchi et al. 2011a,b, 2013a, respectively). We used a Kolmogorov-Smirnov (K-S) test to check the statistical significance of the differences in the observed distributions. The test yields a confidence level exceeding $3 \sigma$ that the two groups of stars have different radial distributions, supporting the hypothesis that the two classes of PMS objects belong indeed to different generations of stars.

\section{Mass accretion rates}

The $\Delta(\mathrm{H} \alpha)$ parameter defined in Sect. 4 allows us to easily derive the $\mathrm{H} \alpha$ emission line luminosity $L(\mathrm{H} \alpha)$ for our bona fide PMS stars. As explained in DM10, $L(\mathrm{H} \alpha)$ is obtained from $\Delta(\mathrm{H} \alpha)$, from the photometric zero point and absolute sensitivity of the instrumental set-up and from the distance to the sources. We have assumed the $\mathrm{Zp}(\mathrm{H} \alpha)=21.63$ and a distance modulus $(m-M)_{0}=12.3$, as mentioned before. The median $L(\mathrm{H} \alpha)$ in our sample is $\sim 9 \times 10^{-3} L^{\odot}$ with the 17 and 83 percentile levels in the distribution at $0.006 L^{\odot}$ and $0.018 L^{\odot}$, respectively.

Following DM10, we derived the accretion luminosity $L_{\text {acc }}$ from the measured value of $\mathrm{L}(\mathrm{H} \alpha)$. In that work it is shown that the ratio $L_{\mathrm{acc}} / L(\mathrm{H} \alpha)$ can be assumed to be constant and, from an elementary fit to the data in the compilation of Dahm (2008) of PMS stars in the Taurus-Auriga association, it is found that $\log L_{\mathrm{acc}}=(1.72 \pm 0.25)+\log L(\mathrm{H} \alpha)$. The median value of $L_{\mathrm{acc}}$ that we find for the PMS stars in $\operatorname{Tr} 14$ is $\sim 0.3 L_{\odot}$.

The mass accretion rate $\dot{M}_{\text {acc }}$ is in turn related to $L_{\text {acc }}$ via the free-fall equation, linking the luminosity released by the impact of the accretion flow with the rate of mass accretion, according to the relationship

$L_{\mathrm{acc}}=\frac{G M_{*} \dot{M}_{\mathrm{acc}}}{R_{*}}\left(1-\frac{R_{*}}{R_{\mathrm{in}}}\right)$,

where $G$ is the gravitational constant, $M_{*}$ and $R_{*}$ the stellar mass and photospheric radius derived in Sect. 5, while $R_{\text {in }}$ is the inner radius of the accretion disc. The latter is uncertain and depends on the exact coupling between the accretion disc and the magnetic field of the star. Following Gullbring et al. (1998), we assume $R_{\text {in }}=5 R_{*}$ for all PMS objects.

The values of $\dot{M}_{\text {acc }}$ derived in this way for the PMS stars in Tr 14 are shown as black triangles in Fig. 11 as a function of the stellar age. The solid line shows the temporal decline of $\dot{M}_{\text {acc }}$ for the evolution of viscous discs, as predicted by Hartmann et al. (1998) for stars of mass $\sim 0.4 M_{\odot}$. It is clear that the distribution of $\dot{M}_{\text {acc }}$ for the PMS objects in our sample is much higher compared to the theoretical predictions. Da Rio et al. (2014) have shown that correlated uncertainties between $\dot{M}_{\text {acc }}$ and the estimated parameters of young stars (such as luminosity, effective temperature, mass, and age) can introduce biases on the apparent decay of $\dot{M}_{\text {acc }}$ as a function of age. More generally, diagrams like those shown in Fig. 11 can be used to study the temporal evolution of $\dot{M}_{\text {acc }}$ only if all stars, even the older ones, were formed under similar conditions. To avoid these possible sources of uncertainty, we concentrate on stars of similar age ( $2 \mathrm{Myr})$ which are numerous in $\operatorname{Tr} 14$ and in the other cluster to which we will compare it later $(\operatorname{Tr} 37)$. The mean value of $\dot{M}_{\text {acc }}$ at an age of 
G. Beccari et al.: Mass accretion rates from multiband photometry in the Carina Nebula: the case of Trumpler 14

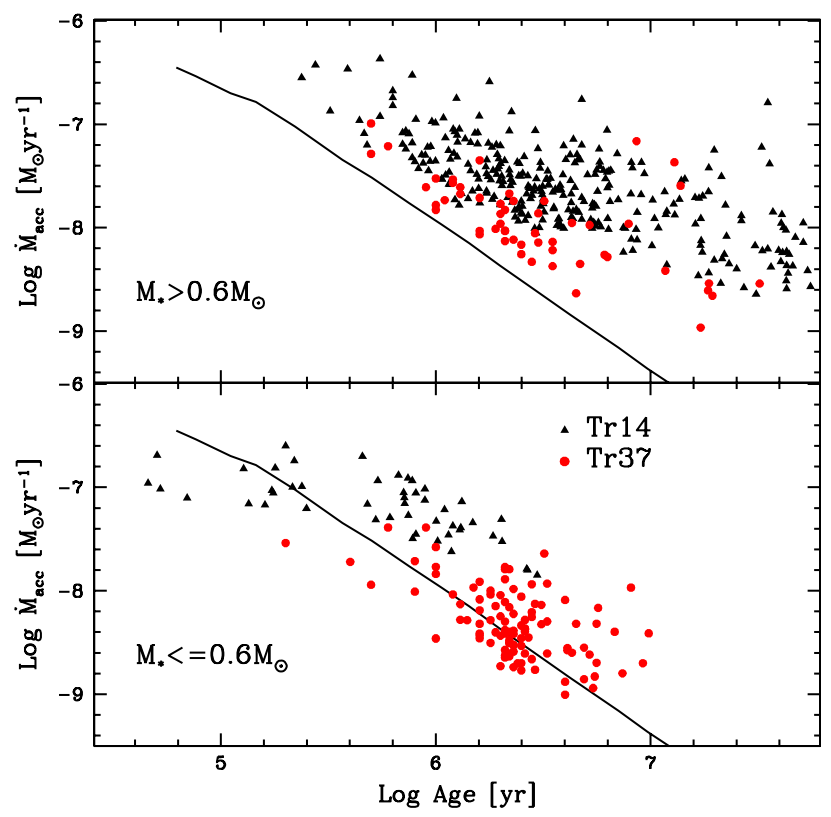

Fig. 11. Derived mass accretion rates as a function of the age of the PMS stars. Stars with masses higher and lower than $0.6 M_{\odot}$ are shown separately. The large black dots are for the stars in $\operatorname{Tr} 14$, whereas the smaller grey dots correspond to objects in $\operatorname{Tr} 37$. The solid line shows the temporal evolution of $\dot{M}_{\text {acc }}$ for viscous discs as predicted by Hartmann et al. (1998).

$\sim 2 \mathrm{Myr}$ is $\sim 1.6 \times 10^{-8} M_{\odot} \mathrm{yr}^{-1}$, while the theoretical model predicts a value of $\sim 5 \times 10^{-9} M_{\odot} \mathrm{yr}^{-1}$.

In order to better understand the origin of this apparent discrepancy we used the photometric catalogue of $\mathrm{T}$ Taury stars belonging to the H II region IC 1396 in Cepheus OB2, including Trumpler 37 ( $\operatorname{Tr} 37)$, published by Barentsen et al. (2011, hereafter B11). In that paper the authors use observations acquired with the Wide Field Camera at the Isaac Newton Telescope (INT) in the $r^{\prime}, g^{\prime}$, and $\mathrm{H} \alpha$ bands, as part of the INT Photometric H-Alpha Survey (IPHAS; Drew et al. 2005). Barentsen et al. (2011) used an observational strategy very similar to the one used in this paper to identify PMS stars from their $\mathrm{H} \alpha$ excess emission and to study their physical parameters and $\dot{M}_{\text {acc }}$. We retrieved their catalogue of $r^{\prime}, i^{\prime}$, and $\mathrm{H} \alpha$ magnitudes, which also contains the masses and radii derived for these objects. We followed the approach described above to determine $E W(\mathrm{H} \alpha)$, $L(\mathrm{H} \alpha), L_{\mathrm{acc}}$, and $\dot{M}_{\mathrm{acc}}$ for the stars in the B11 catalogue. The values of $\dot{M}_{\text {acc }}$ found in this way are shown as red dots in Fig. 11. The values are in full agreement with those found by B11 (see their Fig. 16), confirming the validity of our approach.

From the results shown in Fig. 11 it is clear that the $\dot{M}_{\text {acc }}$ of the stars in $\operatorname{Tr} 14$ are on average higher than those measured in $\operatorname{Tr} 37$, which agree more closely with the prediction from the viscous model. In fact, this is only the case for the lower mass stars, shown in the lower panel of Fig. 11, while the objects more massive than $0.6 M_{\odot}$ even in $\operatorname{Tr} 37$ have $\dot{M}_{\text {acc }}$ values systematically above those predicted by the models of Hartmann et al. (1998). To better characterize the differences between the stars that we have sampled in the two regions, we compare their mass distributions in Fig. 12.

As already shown in Fig. 9, the mass distribution for the PMS stars in our Tr 14 sample peaks at $\sim 1 M_{\odot}$ and drops at lower masses because of photometric incompleteness (see also solid line in Fig. 12). On the other hand, the catalogue of B11 samples a significantly different range of masses in $\operatorname{Tr} 37$. The histogram (dashed line in Fig. 12) clearly indicates that the majority

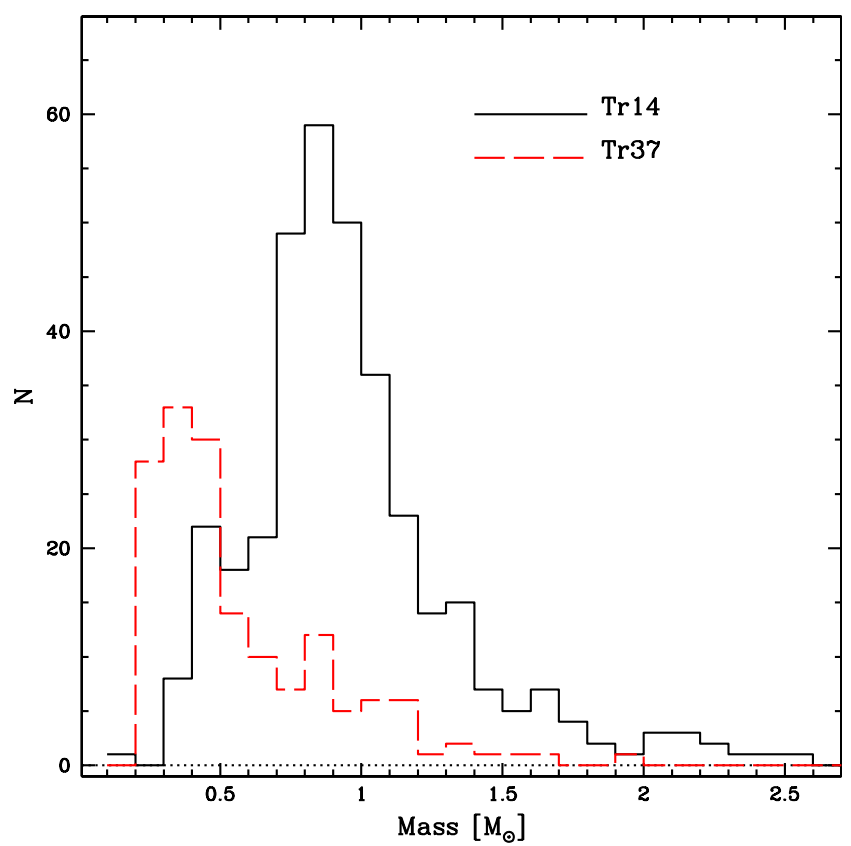

Fig. 12. Histograms showing the mass distributions of the PMS stars in $\operatorname{Tr} 14$ (solid line) and Tr 37 from the catalogue of B11 (dashed line).

of PMS objects in this star forming region have masses from $\sim 0.1 M_{\odot}$ to $\sim 0.6 M_{\odot}$, with a peak at $\sim 0.3 M_{\odot}$. Since the mass accretion rate $\dot{M}_{\text {acc }}$ scales approximately linearly with the mass of the objects (e.g. De Marchi et al. 2011a, 2013a; Barentsen et al. 2011; Spezzi et al. 2012), the large difference in the sampled masses in the two datasets is a plausible explanation for the difference in the observed distribution of $\dot{M}_{\text {acc }}$.

\section{Spectral energy distributions}

By combining the WFI optical photometry with the SOFI and Spitzer/IRAC datasets described in Sect. 2, we are able to derive the SEDs of the stars in the central $\sim 5^{\prime}$ around the cluster centre (see Fig. 1). The flux at each wavelength has been corrected for extinction, according to the reddening values derived in Sect. 3 for the individual objects.

Of the 389 bona fide PMS stars, 57 fall outside the area covered by the other photometric catalogues, while 227 PMS have at least a SOFI and/or Spitzer counterpart. We find these numbers quite reasonable, given that both the SOFI and Spitzer observations are considerably shallower than the WFI images and do not allow the lowest mass PMS stars observed in the optical bands to be detected. Moreover, the lower angular resolution of the near-IR observations further reduces the detection limits of the Spitzer and SOFI data. Nevertheless, the available data allow us for the first time to collect a sample of 227 SEDs of bona fide PMS stars in $\operatorname{Tr} 14$, some of which are shown in Fig. 13. We note that the error bars in the figure are smaller than the symbol size because of the low flux uncertainties, and therefore are not shown.

Over-plotted on the observed SED of each star (large dots), we show a synthetic spectrum (grey line) from the NextGen stellar atmosphere models (Allard et al. 2012) for the specific stellar parameters that we have derived in Sect. 5. We stress here that the models are not fitted to the observed SEDs, but are directly calculated for the measured stellar parameters from our photometry. 

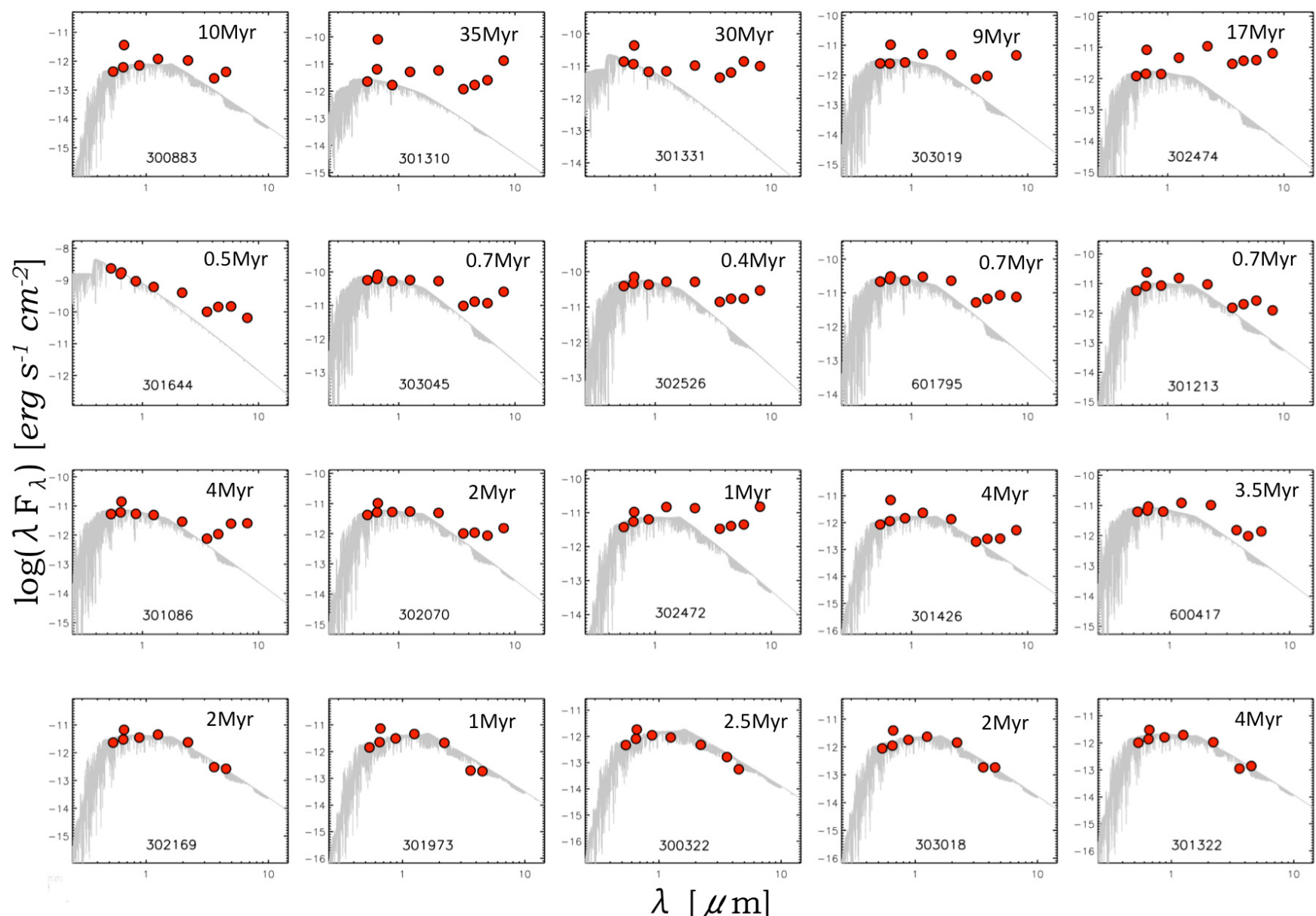

Fig. 13. SEDs of a sample of 20 bona fide PMS objects labelled with the IDs given in the photometric catalogue. The age of each object is also given. The extinction corrected fluxes are represented by the large dots. The error bars are smaller than the symbols and as such are not shown. In grey we show the NextGen stellar models that correspond to the physical parameter of each star, as derived in Sect. 5.

The five SEDs in the top row of Fig. 13 are for older PMS objects, while those shown in the second and third row are for PMS stars with ages younger than 5 Myr. Finally, the bottom row shows the SEDs for five bona fide PMS stars that have excess $\mathrm{H} \alpha$ emission but no IR excess at the wavelengths covered by these observations. A visual inspection reveals that there is an excellent agreement between the atmospheric models and the observed SEDs.

It has been convincingly shown that the SEDs can be efficiently used to detect discs around PMS objects and to study their nature and evolution (see e.g. Espaillat et al. 2010; Fang et al. 2013; Merín et al. 2010, and references therein). Moreover, the slope of the SED in the wavelength region covered by the near-IR excess, i.e. the spectral index $\alpha$, can be used to characterize different object classes (Lada et al. 2006; see also Alcalá et al. 2008 and references therein). However, we stress that in the present work we are mainly interested in using the SEDs in order to identify stars with near-IR excess. Among the 227 PMS with an IR detection (either SOFI, Spitzer, or both), 82 (i.e. 36\%) show near-IR excess at the sampled wavelengths. Only $\sim 10 \%$ of them have ages older than $10 \mathrm{Myr}$, in relatively good agreement with the predictions made so far on the lifetime of accreting protoplanetary discs and based mainly on spectroscopic studies of nearby starforming regions (e.g. Fedele et al. 2010). Finally, it is interesting to note that $\sim 40 \%$ and $\sim 47 \%$ of the young and old
PMS objects, respectively, show IR excess, i.e. show a disc at the sampled IR wavelength.

At first sight, the number of $\mathrm{H} \alpha$ excess emitters detected in our optical images that also show an excess in the SOFI and Spitzer images might seem low. It is important to note, however, that the observed SEDs in the near-IR depend on the opacity of the disc, which is governed by the dust. In particular, dust opacity is affected by the composition of the dust and by grain growth and settling. For example Espaillat et al. (2012) showed that, for the same dust-to-gas mass ratio, discs with small grain size are more flared than discs with large ones. However, the detection limit of the SOFI and Spitzer images and the lack of observations at wavelengths longer than $10 \mu \mathrm{m}$ do not allow us to investigate in detail the nature and the physical state of the discs in most of the bona fide PMS stars detected in this work.

It is possible that most of the older PMS stars in our sample should be classified as transitional phase characterized by dust removal in the inner regions resulting in a lack of infrared flux. This deficiency has become the defining characteristic of transitional discs (TDs Merín et al. 2010). Our results would imply a scenario in which a transitional disc still hosts a significant amount of gas accreting onto the star, even at ages older than 10 Myr. Similar results were already obtained by Zuckerman et al. (1995) but interestingly, Manara et al. (2014) recently published a spectroscopic study of the gas content, accretion, and 
wind properties of a sample of 22 TDs with X-Shooter. They find that $80 \%$ of the TDs in their sample show $\dot{M}_{\text {acc }}$ compatible with those of classical TTauri obects. Moreover, they conclude that in their somewhat limited sample of TDs there is a gas rich inner disc with density similar to that of discs in classic PMSs. Bergin et al. (2013), using observations from the Herschel Space Observatory PACS Spectrometer, found that the old ( 10 Myr) PMS star TW Hydrae is surrounded by a gas disc with mass exceeding $0.05 M_{\odot}$. This surprising result indicates that TW Hydrae is an old PMS star containing a massive gas disc that is several times the minimum mass required to make all the planets in our solar system. Even more recently, Roberge et al. (2013) and Moór et al. (2013) reported the discoveries of a low-mass protoplanetary-like gaseous disc, together with debris discs, around the older PMS stars 49 Ceti and HD 95086, with ages ranging from $10 \mathrm{Myr}$ to $30 \mathrm{Myr}$. All these results support a scenario in which old and evolved debris discs still host a significant amount of gas and confirm our results with completely independent measurements.

\section{Summary}

In this work we present optical observations of PMS stars in the cluster Tr 14 in the Carina Nebula, obtained with the WFI imager at La Silla. We further combine these optical observations with near-IR observations made with SOFI and Spitzer in order to provide a more complete description of the cluster's young stellar populations. The main results can be summarized as follows:

1. We present the deepest optical CMD to date for this cluster.

2. Using $\mathrm{H} \alpha$ photometry we are able to identify 356 bona fide PMS stars showing $\mathrm{H} \alpha$ excess emission with $E W(\mathrm{H} \alpha)>$ $20 \AA$. We interpret this observational feature as an indication that these objects are still actively accreting gas from a circumstellar disc.

3. From the comparison of the HR diagram with PMS evolutionary models we derive ages and masses of the PMS stars. We find that most of the PMS objects are younger than 10 Myr with a median age of 2 Myr. Surprisingly, we find that $\sim 20 \%$ of the mass accreting objects are older than $8 \mathrm{Myr}$.

4. The presence of candidate long-lived accreting discs is in line with the findings in a number of Galactic (Beccari et al. 2010; De Marchi et al. 2013b) and extragalactic (Panagia et al. 2000; De Marchi et al. 2010, 2011a,b, 2013a,b; Spezzi et al. 2012) starburst clusters.

5. Using the derived stellar physical parameters (mass, radius) of the PMS objects and their $\mathrm{H} \alpha$ luminosities $L(\mathrm{H} \alpha)$, we derive the accretion luminosity $L_{\text {acc }}$ and mass accretion rates $\dot{M}_{\text {acc }}$. We find that $\dot{M}_{\text {acc }}$ decreases with time, in line with the predictions of models of viscous discs, but the $\dot{M}_{\text {acc }}$ that we measure are systematically higher than those predictions. Taking into account the photometric completeness in the $\mathrm{H} \alpha$ observations and our requirement that a bona fide PMS star be classified as such only if it has $E W(\mathrm{H} \alpha)>20 \AA$, we determine the detection limit to the measured $\dot{M}_{\text {acc }}$ rates. For comparison, we apply the same analysis to PMS stars in $\operatorname{Tr} 37$, where the distribution of PMS masses peaks at $\sim 0.3 M_{\odot}$. We demonstrate that our $\dot{M}_{\text {acc }}$ values are reliable and that it is the mass distribution of the PMS objects sampled in $\operatorname{Tr} 14$ that affects the measured $\dot{M}_{\text {acc }}$. A K-S test demonstrates that, once the same mass range is selected, the $\dot{M}_{\text {acc }}$ distributions in $\operatorname{Tr} 14$ and $\operatorname{Tr} 37$ are indistinguishable from one another.

6. We finally combine the optical WFI photometry with nearIR observations from SOFI and Spitzer, all corrected for extinction, in order to build SEDs for each PMS star in Tr 14. A comparison with synthetic spectra, calculated using NextGen atmosphere models for the specific physical parameters measured for these stars, indicates that only a small fraction of them have near-IR excess, at any age. The lack of data at wavelengths above $10 \mu \mathrm{m}$ does not allow us to investigate in detail the nature and the physical properties of the discs. However, our analysis suggests the presence of transitional discs in which a large amount of gas is still present and sustains accretion onto the PMS object at ages older than $10 \mathrm{Myr}$. This scenario is supported by the recent discoveries with Herschel of transitional discs containing a massive gas component around the relatively old PMS stars TW Hydrae, 49 Ceti, and HD 95086. Our results are in full agreement with these findings and taken together they challenge the conventional wisdom of circumstellar disc evolution, possibly implying a new scenario for the planet formation mechanism. The existence of a population of old discs suggests that the planet formation process could proceed on much longer timescales than previously thought.

Acknowledgements. We wish to thank the anonymous referee for insightful comments that have helped to improve the presentation of our work. This research has made use of the NASA/IPAC Infrared Science Archive, which is operated by the Jet Propulsion Laboratory, California Institute of Technology, under contract with the National Aeronautics and Space Administration. N.P. acknowledges partial support by STScIDDRF grant D0001.82435. M.Z. was supported by Proyecto Fondecyt Regular 1110393, The BASAL Center for Astrophysics and Associated Technologies PFB-06, and from the Ministry of Economy, Development, and Tourism's Iniciativa Cientfica Milenio through grant IC12009, awarded to the Millennium Institute of Astrophysics MAS.

\section{References}

Allard, F., Homeier, D., \& Freytag, B. 2012, Roy. Soc. London Philos. Trans. Ser. A, 370, 2765

Appenzeller, I., \& Mundt, R. 1989, A\&ARv, 1, 291

Ascenso, J., Alves, J., Vicente, S., \& Lago, M. T. V. T. 2007, A\&A, 476, 199

Baraffe, I., Chabrier, G., \& Gallardo, J. 2009, ApJ, 702, L27

Barentsen, G., Vink, J. S., Drew, J. E., et al. 2011, MNRAS, 415, 103

Beccari, G., Spezzi, L., De Marchi, G., et al. 2010, ApJ, 720, 1108

Beccari, G., De Marchi, G., Panagia, N., \& Pasquini, L. 2014, MNRAS, 437, 2621

Bellazzini, M., Fusi Pecci, F., Messineo, M., Monaco, L., \& Rood, R. T. 2002, AJ, 123, 1509

Bergin, E. A., Cleeves, L. I., Gorti, U., et al. 2013, Nature, 493, 644

Bertout, C. 1989, ARA\&A, 27, 351

Bessell, M. S., Castelli, F., \& Plez, B. 1998, A\&A, 333, 231

Calvet, N., Hartmann, L., Strom, E. 2000, in Protostars and Planets, eds V. Mannings, A. Boss, \& S. Russell (Tucson: University of Arizona Press), 377 Carraro, G., Romaniello, M., Ventura, P., \& Patat, F. 2004, A\&A, 418, 525

Da Rio, N., Robberto, M., Soderblom, D. R., et al. 2009, ApJ, 183, 261

Da Rio, N., Gouliermis, D. A., \& Gennaro, M. 2010, ApJ, 723, 166

Da Rio, N., Jeffries, R. D., Manara, C. F., \& Robberto, M. 2014, MNRAS, 439, 3308

Degl'Innocenti, S., Prada Moroni, P. G., Marconi, M., \& Ruoppo, A. 2008, Ap\&SS, 316, 25

De Marchi, G., Panagia, N., \& Romaniello, M. 2010, ApJ, 715, 1

De Marchi, G., Panagia, N., Romaniello, M., et al. 2011a, ApJ, 740, 11

De Marchi, G., Paresce, F., Panagia, N., et al. 2011b, ApJ, 739, 27

De Marchi, G., Beccari, G., Panagia, N. 2013a, ApJ, 775, 68

De Marchi, G., Panagia, N., Guarcello, M. G., \& Bonito, R. 2013b, MNRAS, 435,3058

Dahm, S. 2008, AJ, 136, 521

Espaillat, C., D’Alessio, P., Hernández, J., et al. 2010, ApJ, 717, 441

Espaillat, C., Ingleby, L., Hernández, J., et al. 2012, ApJ, 747, 103

Fang, M., Kim, J. S., van Boekel, R., et al. 2013, ApJs, 207, 5

Fedele, D., van den Ancker, M. E., Henning, T., Jayawardhana, R., \& Oliveira, J. M. 2010, A\&A, 510, A72

Fender, R. P., Russell, D. M., Knigge, C., et al. 2009, MNRAS, 393, 1608 
Gullbring, E., Hartmann, L., Briceño, C., \& Calvet, N. 1998, ApJ, 492, 323 Hartmann, L., Calvet, P., Gullbring, E., \& DAlessio, P. 1998, ApJ, 495, 385 Herbst, W., Bailer-Jones, C. A. L., Mundt, R., Meisenheimer, K., \& Wackermann, R. 2002, A\&A, 396, 513

Hora, J. L., Carey, S., Surace, J., et al. 2008, PASP, 120, 1233

Hur, H., Sung, H., \& Bessell, M. S. 2012, AJ, 143, 41

Koenigl, A. 1991, ApJ, 370, L39

Lada, C. J., Muench, A. A., Luhman, K. L., et al. 2006, AJ, 131, 1574

Lidman, C., \& Cuby, J. C. 2000, SOFI user's manual, Ref. LSO-MAN-ESO40100-0004 (Garching: ESO)

Lynden-Bell, D., \& Pringle, J. E. 1974, MNRAS, 168, 603

Manara, C. F., Testi, L., Natta, A., et al. 2014, A\&A, 568, A18

Marigo, P., Girardi, L., Bressan, A., et al. 2008, A\&A, 482, 883

Merín, B., Brown, J. M., Oliveira, I., et al. 2010, ApJ, 718, 1200

Mihalas, D., \& Binney, J. 1981, Galactic Astronomy: Structure and Kinematics, 2nd. edn. (San Francisco: Freeman)

Moór, A., Juhász, A., \& Kóspál, Á., et al. 2013, ApJ, 777, L25

Panagia, N., Romaniello, M., Scuderi, S., \& Kirshner, R. 2000, ApJ, 539, 197

Preibisch, T. 2012, RA\&A 12,
Rigliaco, E., Natta, A., Randich, S., Testi, L., \& Biazzo, K. 2011, A\&A, 525, A47

Roberge, A., Kamp, I., Montesinos, B., et al. 2013, ApJ, 771, 69

Romaniello, M. 1998, Ph.D. Thesis, Scuola Normale Superiore, Pisa, Italy

Romaniello, M., Panagia, N., Scuderi, S., \& Kirshner, R. P. 2002, AJ, 123, 915

Sana, H., Momany, Y., Gieles, M., et al. 2010, A\&A, 515, A26

Shu, F. H., Najita, J., Ruden, S. P., \& Lizano, S. 1994, ApJ, 429, 797

Soderblom, D. R., Hillenbrand, L. A., Jeffries, R. D., Mamajek, E. E., \& Naylor,

T. 2013, Protostars and Planets VI (University of Arizona Press), accepted [arXiv: 1311.7024]

Spezzi, L., de Marchi, G., Panagia, N., Sicilia-Aguilar, A., \& Ercolano, B. 2012, MNRAS, 421,78

Stetson, P. B. 1987, PASP, 99, 191

Stetson, P. B., 1994, PASP, 106, 250

Tognelli, E., Degl'Innocenti, S., \& Prada Moroni, P. G. 2012, A\&A, 548, A41

Vazquez, R. A., Baume, G., Feinstein, A., \& Prado, P. 1996, A\&AS, 116, 75

Weidner, C., Kroupa, P., \& Maschberger, T. 2009, MNRAS, 393, 663

White, R. J., \& Basri, G. 2003, ApJ, 582, 1109

Zuckerman, B., Forveille, T., \& Kastner, J. H. 1995, Nature, 373, 494 